\title{
THEORIES OF HYDROPHOBIC EFFECTS AND THE DESCRIPTION OF FREE VOLUME IN COMPLEX LIQUIDS
}

\author{
LAWRENCE R. PRATT, SHEKHAR GARDE, AND GERHARD HUMMER \\ Theoretical Division, Los Alamos National Laboratory \\ Los Alamos, New Mexico 87545 USA
}

\begin{abstract}
Recent progress on molecular theories of hydration of nonpolar solutes in aqueous solution has led to new ways of thinking about the old issue of free volume in liquids. This article surveys the principal new results with particular attention to general issues of packing in liquids.
\end{abstract}

\section{Introduction}

Aqueous solutions of colloidal solutes are preeminent examples of complex liquids. In such settings, attention is often directed towards the issues of macromolecular structure, aggregation, and dynamics. However, one aspect of these problems is stubbornly associated with the small size scale of molecules, in particular the small size of a water molecule. That problem is the molecular understanding and description of hydration effects hydrophobic effects - that stabilize membranes, micelles, folded proteins, and aggregates of such structures. This presentation will focus on that basic, molecular scale issue underlying aqueous solutions of interest both to biophysics and material science of colloids.

Because hydrophobic effects are so broadly discussed, it might be surprising to notice that workers on molecular theories of hydrophobic effects have not achieved good agreement on that molecular theory, at least when we proceed beyond the primitive stage of the principles of statistical mechanics and specification of the intermolecular interactions involved. The empirical fact is that different researchers hold different opinions on the correctness of several available theories, each of which agrees with a set of experimental data at least roughly. A sampling of different perspectives is 
available from the references [1-33]; this collection is not intended to be complete, however.

Furthermore, the simplest extensions of our experimental information can spark new debates about our understanding of hydrophobic effects.

A current example, the effect of pressure on hydrophobic stabilization of folded proteins, is discussed below.

In consequence of such observations, the theory surveyed here was designed for maximal simplicity on the fundamentals of statistical mechanics and on the physical assumptions applied to the particular problems. The theory that was developed is relevant to the traditional packing problems of theories of liquids and we emphasize that connection in the discussion here.

A central problem for the theory of hydrophobic effects is the old, but imperfectly solved, theoretical problem of finding space for a solute in liquid solvents. As is well recognized, liquids are dense, disordered materials. It is this combination of attributes that makes these problems difficult. The description of free volume in hard core model liquids is central to the modern understanding of the van der Waals equation of state and is basic to such ordering phase transitions as the hard sphere freezing and the liquid crystal phase transitions [34]. In the specific motivating case considered here, the particular molecular structuring characteristic of liquid water is expected to be important. So we must preserve the fidelity of the description of the structure of liquid water, in addition to tackling the problem of packing in dense, disordered materials.

This lecture develops a new conceptualization of this old problem and new techniques for predicting the fractional free volume accessible to hard core molecules in condensed phases. This new approach is based upon an information theory perspective that has general applicability and was initially explicitly heuristic. Information was sought on a condensed medium of interest and on that basis a prediction of the fractional free volume accessible to a hard model solute was made. For some important cases considered so far, the detail of information required for accurate, interesting predictions has been surprisingly modest.

\section{Free Volume Statistics}

Consider the solubility of inert gases in aqueous solutions. The medium is liquid water and the solute is idealized as a hard object, perfectly repelling the center (oxygen atom) of each water molecule. For such models the interaction part of the chemical potential of the solute is obtained as

$$
\beta \Delta \mu=-\ln p_{0},
$$


with $\mathrm{p}_{0}$ the probability that the hard solute could be inserted into the system without overlap of van der Waals volume of the solvent; $1 / \beta=\mathrm{k}_{B} \mathrm{~T}$. This is a specialization of Widom's formula [35, 36]

$$
\exp \{-\beta \Delta \mu\}=\langle\exp \{-\beta \Delta U\}\rangle_{0} .
$$

$\Delta U$ is the change in the solute-solvent interaction potential energy upon placement of the solute in an arbitrary position in the solvent and the average indicated by $\langle\ldots\rangle_{0}$ is over the thermal motion of the solvent unaffected by the solute. The solute is a test particle for this calculation. For the hard core model being considered, $\Delta U$ is either zero or infinity, so the average sought involves a random variable with value either one or zero; the averaging collects the fraction of solute placements that would be allowed. If presented with a thermal configuration of a large volume of solvent, we might estimate these quantities by performing many trial placements of the solute throughout the solvent and determining the fraction of those trial placements that would be allowed. This estimates $V_{\text {free }} / V$, the fractional free volume accessible to the solute. Thus, Eq. (1) is a free volume formula [37], exact for the model being considered.

The operation of these formulae can be viewed alternatively: Imagine identifying a molecular scale volume at an arbitrary position in the liquid system by (1) hypothetical placement of the solute and (2) determination of those positions of water oxygen atoms that would be excluded due to solute-solvent interactions. We will call this volume the observation volume. With such a molecular scale volume defined we could keep track, say during a simulation calculation, of the probabilities $\mathrm{p}_{n}$ that $n=0,1, \ldots$ oxygen atom occupants are observed. As the notation suggests, $\mathrm{p}_{0}$ is the probability that no occupants are observed in the molecular volume.

Our strategy for predicting of $\mathrm{p}_{0}$ will be to model the distribution $\mathrm{p}_{n}$ and to extract the extreme value $\mathrm{p}_{0}$. This is a primitive approach to theories of $\beta \Delta \mu$ and solubilities of inert gases water. Both more and less subtle theoretical works on these topics have been long available. "Less subtle" here means simulation calculations, techniques more straightforwardly useful than many "more subtle" approaches. The "more subtle" means here that further statistical quantities have been introduced for spherical solutes with the intention that they might facilitate more expansive approximate theories. These include

$$
\begin{gathered}
d_{1}(\lambda)=-\frac{d p_{0}(\lambda)}{d \lambda}, \\
G(\lambda)=\left(\frac{-1}{4 \pi \lambda^{2} \rho}\right) \frac{d \ln p_{0}(\lambda)}{d \lambda} .
\end{gathered}
$$


$\lambda$ is the radius of a center-to-center exclusion sphere. These quantities have been useful in suggesting physical theories because they have interpretations that are appreciated physically. $d_{1}(\lambda)$, is the distribution function of distances $\lambda$ from an arbitrary point in the liquid to the nearest solvent center. $4 \pi \lambda^{2} \rho G(\lambda)$ [Eq. (4)] is, in view of Eq. (1), the derivative with respect to exclusion radius of the hydration free energy due to intermolecular interactions, in thermal energy units $\mathrm{k}_{B} \mathrm{~T}$. It gives the compressive force exerted by the solvent on the hard spherical solute. In addition, $4 \pi \lambda^{2} \rho G(\lambda) d \lambda$ is the expected number of solvent centers in a shell of radius $\lambda$ and width $d \lambda$ outside a hard sphere that excludes solvent centers from a ball of radius $\lambda$.

\section{Simulation Results for Liquid Water}

Some of the simulation work has determined the quantities of Eqs. (3) and (4) for molecular liquids represented realistically at the current state-ofthe-art $[14,16]$. Thus we know that $d_{1}(\lambda)$ for liquid water and for liquid nhexane are both unimodal with maximum displaced by a distance less than $0.1 \AA$; the maximum occurs at slightly smaller distances for liquid n-hexane than for liquid water $[14,16]$. This difference in the most probable cavity size between liquid n-hexane and liquid water is not large. The difference in the most probable cavity size between liquid water and a reference random medium, with sites of the same radius and distributed randomly at the water density, is greater than the difference between n-hexane and water. So the differences observed between the two molecular liquids considered are not purely reflections of molecular size and density. This comparison addresses the idea that the low solubility of inert gases in liquid water might be due to the small size of the water molecule and the possibility that "interstitial" cavities would, on this basis, be smaller in water than in the organic liquid $[3,38]$. The fact that these differences between water and n-hexane are slight is associated with the fact that the basic units considered in n-hexane are the methyl and methylene groups. These are not so different in size from a water molecule. It should be noted also that on a packing fraction basis, typical organic liquids are denser than liquid water [6].

The notable distinction between the results for liquid water and liquid n-hexane is that the distribution $d_{1}(\lambda)$ is narrower for liquid water. This suggests that the liquid water phase is less flexible than the liquid n-hexane phase in opening cavities of substantial size.

Simulation calculations have also produced $G(\lambda)$ for $0<\lambda<3.0 \AA$, approximately. This size range covers the simplest atomic solutes $\mathrm{He}$ and $\mathrm{Ne}$ but not much more. This does, however, permit comparison between water and organic solvents, and it permits comparison of available theories with 
the simulation data. Thus for the range $2.0 \AA<\lambda<3.0 \AA, G(\lambda)$ for liquid water is approximately two-times larger than for n-hexane. Water exerts a higher compressive force on the surface of an inert solute than do typical organic liquids; water squeezes-out hydrophobic solutes [39].

The checking of theories against the available simulation data $G(\lambda)$ for water has also been revealing $[14,16]$. We now know that the predictions of the scaled particle model $[40,41]$ are significantly below the numerically exact results for $G(\lambda)$. The Pratt-Chandler (PC) integral equation theory [42-44] predicts results for $G(\lambda)$ that are significantly too large. The more pragmatic revised scaled particle model due to Stillinger [2] typically predicts $G(\lambda)$ between those two theories and with some empiricism about interpolation junctions can describe the available simulation data satisfactorily $[14,16]$. For sizes $\lambda \gg 3.0 \AA$ the available computer simulation data are less extensive, the theories less convincing, and the checking has been pursued less vigorously. See, however, the recent results of Reference [45].

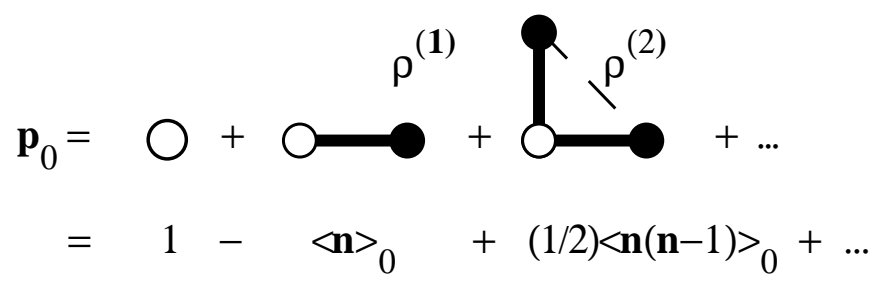

Figure 1. Mayer-Montroll expansion for the insertion probability $\mathrm{p}_{0}$. The standard notation follows [50, 51], e.g., the solid bonds indicate Mayer f-functions. The second line gives the evaluations for the diagrams shown in the case of a hard core solute. $n$ is the number of solvent centers in the observation volume.

\section{Information Model}

We now return to the theoretical program of predicting $\mathrm{p}_{0}$. What are the standard theoretical tools for this? The most immediate guiding theory is the 'inclusion-exclusion' development [46] of Eq. (2) [47, 48, 49]:

$$
p_{0}=1+\sum_{m=1}^{\infty} \frac{(-1)^{m}}{m !} \int_{v} d \mathbf{r}_{1} \int_{v} d \mathbf{r}_{2} \cdots \int_{v} d \mathbf{r}_{m} \rho^{(m)}\left(\mathbf{r}_{1}, \mathbf{r}_{2}, \ldots, \mathbf{r}_{m}\right)
$$

where $\rho^{(m)}$ is the $m$-body joint density for solvent centers. This is depicted in a standard way in Figure 1. These are standard combinatorial results, frequently seen in forms such as [46]

$$
p_{0}=1-\langle n\rangle_{0}+\sum_{m=2}^{\infty} \frac{(-1)^{m}}{m !}\langle n(n-1) \cdots(n-m+1)\rangle_{0} \text {. }
$$




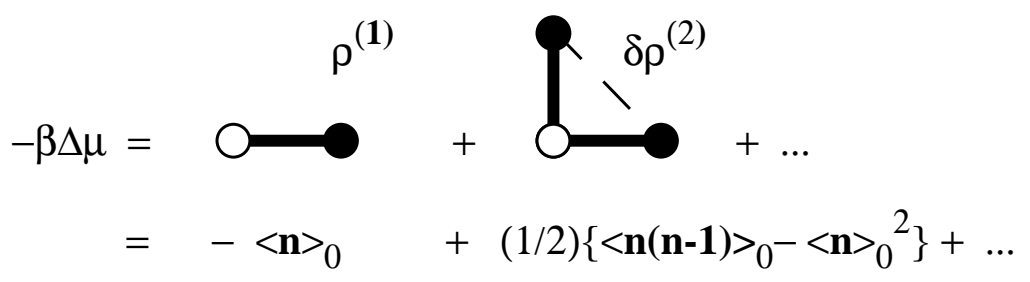

Figure 2. Virial expansion, notation as in Figure 1. Note that 'virial' often connotes a low density expansion but here we are assuming full knowledge of the medium correlation functions in the absence of the solute; $\delta \rho^{(2)}(1,2)=\rho^{(2)}(1,2)-\rho^{(2)}(1) \rho^{(1)}(2)$. Thus from the perspective of a density expansion this organization shuffles the contributions to the virial coefficients. In fact, here contributions are ordered according to the number of bonds attached to the root point. Successive contributions have a structure that may be derived from that of familiar cumulants with the formal replacement $\left\langle n^{k}\right\rangle_{0} \rightarrow\langle n(n-1) \cdots(n-k+1)\rangle_{0}$; then $\mathrm{p}_{0}$ can be formally expressed as $\left\langle e^{-n}\right\rangle_{0}$. See $[52,53,54]$. Table 1 gives formulae for contributions through 5 th order.

Here the random variable $n$ is the number of solvent centers within the observation volume and, e.g., $\langle n\rangle_{0}$ is the expected number of centers within the observation volume. Several important points can be made from Figure 1. The first is that the van der Waals approximation, the primordial free volume model, is obtained from the first two terms shown $\beta \Delta \mu \approx-\ln \left[1-\langle n\rangle_{0}\right]$. The second point is direct and basic: $\mathrm{p}_{0}$ is naturally expressed in terms of occupancy moments, indeed binomial moments here. The sum truncates sharply for cases where a finite maximum number of particles can be present in the observation volume. The sum can be of practical value in the van der Waals case where only the first nontrivial term is retained. For large solute volumes or solvent densities, this sum is not directly useful; we seek a way to exploit the same information but in a more broadly useful form.

The next most immediate theoretical guidance comes from the virial expansion depicted in Figure 2. This can be considered a resummation of the series Figure 1 and is better in the sense that such a truncation cannot produce a negative probability as truncation of Figure 1 can do. However, when that trouble is avoided Figure 2 can still be less compact and truncations can be less accurate. For example, the van der Waals, single term approximation to Figure 1 can be satisfactory and then Figure 2 with a single term is likely to be less so.

Ultimately these considerations avoid the issue that we have only limited information and we want to make the best prediction of $\mathrm{p}_{0}$ that we can. When the problem is stated this way what to do next is clear: we model the probabilities $p_{n}$ on an information theory basis. We consider a relative 
TABLE 1. Successive contributions to the series Figure 2 . $\left(\begin{array}{c}n \\ j\end{array}\right) \equiv n(n-1) \cdots(n-j+1) / j$ ! is the binomial coefficient.

\begin{tabular}{|c|c|}
\hline Order & Formula \\
\hline 1 & $+\left\langle\left(\begin{array}{l}n \\
1\end{array}\right)\right\rangle_{0}$ \\
\hline 2 & $-\left\langle\left(\begin{array}{l}n \\
2\end{array}\right)\right\rangle_{0}+\left\langle\left(\begin{array}{l}n \\
1\end{array}\right)\right\rangle_{0}^{2} / 2$ \\
\hline 3 & $+\left\langle\left(\begin{array}{l}n \\
3\end{array}\right)\right\rangle_{0}-\left\langle\left(\begin{array}{l}n \\
1\end{array}\right)\right\rangle_{0}\left\langle\left(\begin{array}{l}n \\
2\end{array}\right)\right\rangle_{0}+\left\langle\left(\begin{array}{l}n \\
1\end{array}\right)\right\rangle_{0}{ }^{3} / 3$ \\
\hline 4 & $-\left\langle\left(\begin{array}{c}n \\
4\end{array}\right)\right\rangle_{0}+\left\langle\left(\begin{array}{c}n \\
1\end{array}\right)\right\rangle_{0}\left\langle\left(\begin{array}{c}n \\
3\end{array}\right)\right\rangle_{0}+\left\langle\left(\begin{array}{c}n \\
2\end{array}\right)\right\rangle_{0}{ }^{2} / 2-\left\langle\left(\begin{array}{c}n \\
1\end{array}\right)\right\rangle_{0}{ }^{2}\left\langle\left(\begin{array}{c}n \\
2\end{array}\right)\right\rangle_{0}+\left\langle\left(\begin{array}{c}n \\
1\end{array}\right)\right\rangle_{0}{ }^{4} / 4$ \\
\hline 5 & $\begin{array}{l}+\left\langle\left(\begin{array}{l}n \\
5\end{array}\right)\right\rangle_{0}-\left\langle\left(\begin{array}{l}n \\
1\end{array}\right)\right\rangle_{0}\left\langle\left(\begin{array}{l}n \\
4\end{array}\right)\right\rangle_{0}+\left\langle\left(\begin{array}{l}n \\
1\end{array}\right)\right\rangle_{0}{ }^{2}\left\langle\left(\begin{array}{l}n \\
3\end{array}\right)\right\rangle_{0} \\
\quad-\left\langle\left(\begin{array}{l}n \\
2\end{array}\right)\right\rangle_{0}\left\langle\left(\begin{array}{l}n \\
3\end{array}\right)\right\rangle_{0}+\left\langle\left(\begin{array}{l}n \\
1\end{array}\right)\right\rangle_{0}\left\langle\left(\begin{array}{l}n \\
2\end{array}\right)\right\rangle_{0}{ }^{2}-\left\langle\left(\begin{array}{c}n \\
1\end{array}\right)\right\rangle_{0}{ }^{3}\left\langle\left(\begin{array}{l}n \\
2\end{array}\right)\right\rangle_{0}+\left\langle\left(\begin{array}{l}n \\
1\end{array}\right)\right\rangle_{0}{ }^{5} / 5\end{array}$ \\
\hline
\end{tabular}

or cross information entropy [55],

$$
\eta\left(\left\{p_{n}\right\}\right)=-\sum_{n=0}^{\infty} p_{n} \ln \left(\frac{p_{n}}{\hat{p}_{n}}\right),
$$

where $\hat{p}_{n}$ represents a "default model" chosen heuristically. The moments that enter into the series Figure 2, obtained from simulations if necessary, are the information typically used. We then maximize this information entropy subject to the constraints that the probabilities reproduce the available information. The formal maximization of this entropy gives probabilities

$$
p_{j} \propto \hat{p}_{j} \exp \left(-\sum_{k=1}^{k_{\max }} \zeta_{k}\left(\begin{array}{l}
j \\
k
\end{array}\right)\right)
$$

where the $\zeta_{k}$ are Lagrange multipliers to be adjusted so that the probabilities finally reproduce the information given initially. The machinery for doing this can be developed straightforwardly. For example, the normalization of the probabilities can be deferred at intermediate stages of the calculation. Then the final thermodynamic result can be given in terms of the required normalization factor

$$
\beta \Delta \mu=\ln \sum_{n=0} \frac{\hat{p}_{n}}{\hat{p}_{0}} \exp \left(-\sum_{k=1}^{k_{\max }} \zeta_{k}\left(\begin{array}{l}
n \\
k
\end{array}\right)\right) .
$$

This is suggestive of the calculation of a partition function for a modestsized set of states with effective interactions. The interesting questions then involve the predictions extracted from the $\mathrm{p}_{j}$ for properties other than the given information. In our case, the property of first interest is $\beta \Delta \mu$.

We can make the comforting observation that use of only $\langle n\rangle_{0}$ and the natural default model $\hat{p}_{j} \propto 1 / \mathrm{j}$ ! produces the Poisson distribution as expected; $\zeta_{1}=-\ln \langle n\rangle_{0}$ and $\beta \Delta \mu=\langle n\rangle_{0}$ as would be found by retaining only 
the first term shown in Figure 2. Note that evaluation of the second term there with the Poisson distribution gives zero as it should [53]; $\left\langle\delta n^{2}\right\rangle_{0}=\langle n\rangle_{0}$ for the Poisson distribution.

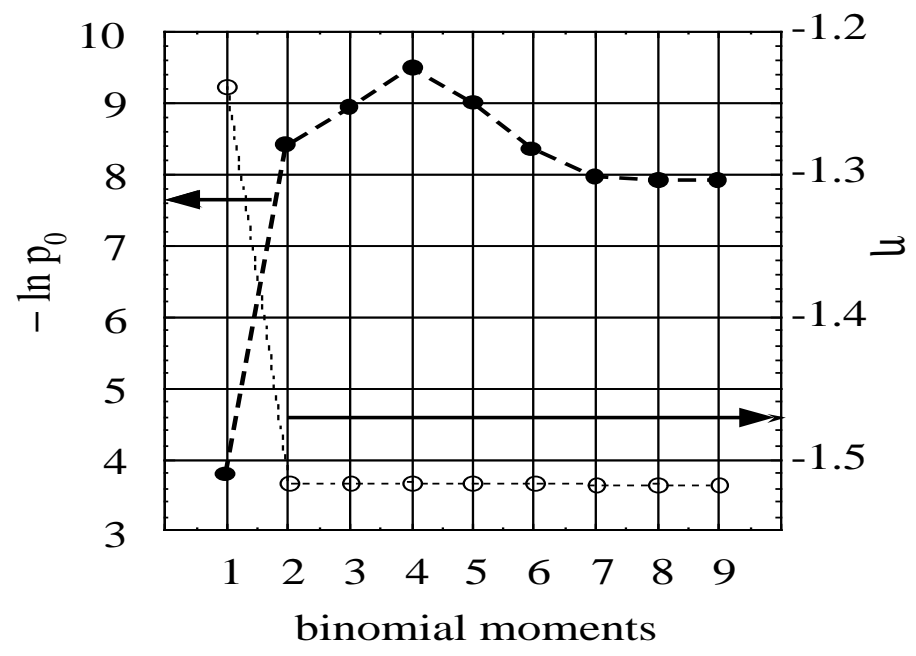

Figure 3. Convergence of predictions for hydration free energy and information entropy with numbers of binomial moments employed; $\hat{p}_{j} \propto 1 / \mathrm{j}$ !. The moment information was obtained from computer simulation of liquid water [58]. $\lambda=3.0 \AA$. The filled (open) circles use the left (right) scale.

Figure 3 shows how this prediction scheme works-out when the solvent is computer simulated liquid water and the solute is a hard sphere of a size appropriate for comparison with a Ne atom. The immediate point is that a model based upon the two moments that might be obtained from experiment, $\langle n\rangle_{0}$ and $\langle n(n-1)\rangle_{0}$, fortuitously provides the most satisfactory simple prediction of $\mathrm{p}_{0}$. The Poisson (one moment) model is not satisfactory. Inclusion of moments higher than the second is not advantageous unless several higher moments are available. The behavior of the information entropy $\eta$ suggests that the initial two moments do a good job of describing the distribution and the subsequent higher moments are 'uninformative.'

These points are further remarkable because recent analyses $[56,57]$ have underscored the fact that Percus-Yevick approximate integral equations can be derived on the assumption that solvent density fluctuations are distributed according to a Gaussian probability functional. The results of the present investigations, including particularly [56-59], give further insight and support to those ideas. The PC approximate integral equation theory of hydrophobic effects [42], at its inception a Percus-Yevick ana- 
logue, can be given a similar basis [56]. The PC theory is thereby given a better foundation than was available at its genesis.

Note that present two-moment model is not precisely an assumption of a Gaussian probability functional for a density field. The occupancies here are required to be nonnegative integers. That this be true of all subvolumes of the observation volume is an important restriction.

TABLE 2. Approximate evaluation of contributions to the series Figure 2 for the circumstances of Figure 3, $\lambda=3.0 \AA$. The column 'Order Contribution' gives approximate values for the formulae of Table 1. 'Cumulative result' is the value obtained for $\beta \Delta \mu$ when the series is truncated at the indicated order. The final row gives the direct result obtained from simulation [58].

\begin{tabular}{cccc}
\hline $\mathrm{j}$ & $\left\langle\left(\begin{array}{l}n \\
j\end{array}\right)\right\rangle_{0}$ & Order Contribution & Cumulative Result \\
1 & 3.77 & 3.77 & 3.77 \\
2 & 5.75 & 1.36 & 5.13 \\
3 & 4.57 & 0.75 & 5.88 \\
4 & 2.04 & 0.50 & 6.38 \\
5 & 0.51 & 0.36 & 6.74 \\
$\infty$ & - & - & 7.93 \\
\hline
\end{tabular}

That specifically binomial moments are involved in the series above emphasizes the point that the occupancies must be nonnegative integers. Further perspective on the convergence issue is obtained by examination of successive contributions to the series Figure 2. Numerical results are shown in Table 2. The first and second order terms make a significant contribution but by themselves are not close to the full answer. The values of the additional terms do not establish a rapid convergence to the known full answer. However, we can view the information theory model as a technique for reorganization of the series.

\subsection{ENTROPY CONVERGENCE}

When the typical occupation numbers $n$ are large the granularity of the distribution $\mathrm{p}_{n}$ is expected to be less significant, at least near the center of the distribution and when viewed on a coarse enough scale. In such circumstances the predictions of two-moment information theory models are not significantly different than those of the PC theory. It is remarkable that a simple calculation along these lines gives a convincing explanation of the puzzling and contentious issue of "entropy convergence" in hydrophobic hydration [60]. 
The phenomenon to be explained is the following: entropies of transfer of non-polar molecules from gas phase or a non-polar solvent into water converge at a temperature of about $400 \mathrm{~K}$ to approximately zero entropy change. Similar behavior was also seen in the microcalorimetry experiments on unfolding of several globular proteins. This behavior is insensitive to the particular hydrophobic solute molecule. Since the entropy is a temperature derivative of a hydration free energy, the convergence temperature identifies a region where graphs of hydration free energy versus temperature are extremal, in fact, maximal. Below that region the hydrophobic hydration free energy increases with temperature but above that region the hydrophobic hydration free energy decreases as the temperature is raised.

The two-moment information theory model above was applied to this problem for hard sphere solutes in water with the heuristic modification that a flat default model was used; $\hat{p}_{j} \propto$ constant for $\mathrm{j} \leq \mathrm{j}_{\max }$ and zero otherwise. This latter adjustment was found empirically to give slightly better hydration free energies. The results of the model and simulation calculations accurately agreed on the temperature dependence of the hydration free energies. To analyze this agreement the information theory model was simplified to a continuous Gaussian distribution that then gives

$$
\begin{aligned}
\Delta \mu & \approx \frac{k_{\mathrm{B}} T}{2}\left\{\frac{\langle n\rangle_{0}^{2}}{\left\langle\delta n^{2}\right\rangle_{0}}+\ln \left[2 \pi\left\langle\delta n^{2}\right\rangle_{0}\right]\right\} \\
& =T \rho_{\text {sat }}(T)^{2}\left\{k_{\mathrm{B}} v^{2} / 2\left\langle\delta n^{2}\right\rangle_{0}\right\}+T\left\{k_{\mathrm{B}} \ln \left(2 \pi\left\langle\delta n^{2}\right\rangle_{0}\right] / 2\right\} .
\end{aligned}
$$

$v$ is the observation volume and $\rho_{\text {sat }}(T)$ is the liquid density along the vapor saturation curve so that $\langle n\rangle_{0}=\rho_{\text {sat }}(T) v$. $\left\langle\delta n^{2}\right\rangle_{0}$ was found to be insensitive to temperature for the relevant conditions. Further, the first term of Eq. (11) is larger than the second. Thus the non-monotonic behavior of the free energy with temperature and the entropy convergence is a consequence of the non-monotonic variation of $T \rho_{\text {sat }}(T)^{2}$ with temperature. The only molecular parameter to complicate matters is the volume $v$ and with this formula $v$ does not affect the entropy convergence temperature [60]. Thus the temperature of entropy convergence is about the same for a wide family of solutes.

The physical point is: the entropy convergence phenomenon occurs for water because of the low and temperature insensitive values of $\left\langle\delta n^{2}\right\rangle_{0}$. In fact, the isothermal compressibility of water at low pressure has a minimum value at $\mathrm{T}=319 \mathrm{~K}$. That temperature differs substantially from the observed entropy convergence temperatures but it is not necessary that these temperatures be approximately equal, just that $\left\langle\delta n^{2}\right\rangle_{0}$ be insensitive to temperature in the region $d\left[T \rho_{\text {sat }}(T)^{2}\right] / d T \approx 0$.

The technical point of Eq. (10) is: this formula is simple and effective but how it is obtained from the series Figure 2 is not simple. Part of the 
complication is that two additional twists have been interjected, the flat default model and the continuous approximation.

\subsection{PRESSURE DENATURATION OF PROTEINS}

It is a common view, based upon our current understanding, that hydrophobic effects provide a nonspecific, cohesive stabilization of compact protein structures. However, it has been argued [62] that our current understanding of hydrophobic effects is not consistent with the experimental facts of pressure denaturation of globular proteins. The information theory model of the previous section was applied also to study hydration free energies and potentials of mean force (pmfs) for two and three hydrophobic spherical solutes in water as a function of pressure [63]. As is well known, those pmfs exhibit contact and solvent-separated minima corresponding, respectively, to cases where the hydrophobic spheres contact each other or where a water molecule intervenes. It was found that increasing pressure shifted the free energy balance of those two cases towards the solvent-separated circumstance. This suggested an intercalation mechanism for pressure denaturation: as the pressure of the liquid is raised, water molecules are forced into protein structure. A similar point of view can be taken of the formation of clathrate hydrates at elevated pressures: at low pressure hydrophobic effects lead to close contacts and to clustering of hydrocarbon gases dissolved in water. Pressure increases stabilize the crystalline phase that eliminates close solute contacts. If attention is focused on the hydrocarbon material this behavior might seem counter-intuitive because the hydrocarbon material seems to expand. But the thermodynamic principle is that increasing pressure stabilizes the phase of lower volume. Therefore, we conclude that the system may be packed more efficiently and have a lower total volume when water molecules are intercalated into the hydrocarbon clusters. These topics will surely be the subject of further research.

\section{Concluding Comments}

Identification of some generalizations and future directions for these theories will provide concluding comments. Firstly, we note that the generalization of these ideas to treat continuous, rather than only hard core repulsive, solute-solvent interactions is known [64].

Secondly, we note the importance in the context of the aqueous solutions of our restriction here to small molecule solutes. It is well recognized that treatment of larger solutes requires consideration of the multiphasic character of these solutions on large length scales [2, 58, 63]. For large enough hard sphere solutes dissolved in water close to phase coexistence, the possibility that the solvent will pull away from the solute surface re- 
quires specific attention. Further subtleties arise when the solute-solvent interactions are not just repulsive but include attractive interactions too $[65,66]$. These issues will surely be the subject of further research in the area of hydrophobic effects. It seems likely that an appropriately designed default model should be able to describe such effects in a physical manner.

Thirdly, we note that these approaches provide some unanticipated answers [64] to questions such as "How is water different from hydrocarbon liquids as a solvent for nonpolar solutes?" The importance of the low and temperature insensitive values of the isothermal compressibility of liquid water is noteworthy. However, such answers are not in the format that is most often intended when such questions are asked. Most often such questions solicit information about particular patterns of solvent structure in the neighborhood of a hydrophobic solutes. Some groundwork has been laid for consideration of those detailed structural issues in a format consistent with the discussion of this paper [67]. Pursuit of answers about the detailed structural issues and their relevance to hydrophobic effects will surely be the subject of future research.

Finally, we note again the relevance of these ideas to the classic problems of packing in liquids. The importance of these issues is reflected in the significance of the hard sphere fluid system to our understanding of liquids. Hard core model systems may not be directly realistic. But it continues to surprise that when attention is directed to new physical problems, e.g. the thermodynamics and structure of glasses or folded proteins, understanding of basic packing problems is again requested. Such problems surface quite broadly [66-72]. It would be interesting to see the ordering phase transitions associated with packing problems, crystallization and liquid crystallization, analyzed on these bases. An initial step along such lines for the hard sphere fluid has been taken [75] but more work is deserved.

We hasten to add that the results so far have not superseded previous theoretical results. But this new approach offers the possibility of better, more physical understandings of packing problems in the equilibrium statistical mechanics of non-crystalline materials and the previous theories of them. This approach has achieved new understanding for the problems of primitive hydrophobic effects. By exploiting information external to conventional theories, even simulation data, and by proposing a pattern for utilizing that information, these approaches begin to respond to Andersen's [76] request for a 'theory of theories.'

\section{Acknowledgement}

This work was supported by the LDRD program at Los Alamos. 


\section{References}

1. A. Ben-Naim and H. L. Friedman. J. Phys. Chem, 71:448-449, 1967.

2. F. H. Stillinger. J. Soln. Chem., 2:141-158, 1973.

3. B. Lee. Biopolymers, 24:813-823, 1985.

4. A. Ben-Naim. J. Chem. Phys., 90:7412-7425, 1989.

5. P. L. Privalov and S. J. Gill. Pure and Applied Chemistry, 61:1097-1104, 1989.

6. A. Pohorille and L. R. Pratt. J. Amer. Chem. Soc., 112:5066-5074, 1990.

7. K. A. Dill. Science, 250:297, 1990.

8. P. L. Privalov, S. J. Gill, and K. P. Murphy. Science, 250:297-298, 1990.

9. K. P. Murphy, P. L. Privalov, and S. J. Gill. Science, 247:559-561, 1990.

10. N. Muller. Acc. Chem. Res., 23:23-28, 1990.

11. L. R. Pratt. Oil and water don't mix. In CLS Division 1991 Annual Review, 5285 Port Royal Rd., Springfield, VA 22161, 1991. National Technical Information Service U. S. Department of Commerce. LA-UR-91-1783.

12. K. A. Sharp, A. Nichols, R. F. Fine, and B. Honig. Science, 252:106-109, 1991.

13. K. A. Sharp, A. Nicholls, R. Friedman, and B. Honig. Biochem., 30:9686-9697, 1991.

14. L. R. Pratt and A. Pohorille. Proc. Natl. Acad. Sci. USA, 89:2995, 1992.

15. T. Lazaridis and M. E. Paulaitis. J. Phys. Chem., 96:3847-3855, 1992.

16. L. R. Pratt and A. Pohorille. Proceedings of the EBSA 1992 International Workshop on Water-Biomolecule Interactions. Societa Italiana di Fisica, Bologna, 1993.

17. B. Lee. Protein Science, 2:733-738, 1993.

18. D. van Belle and S. J. Wodak. J. Am. Chem. Soc., 115:647-652, 1993.

19. A. Ben-Naim and R. M. Mazo. J. Phys. Chem., 97:10829-10834, 1993.

20. A. Holtzer. Biopolymers, 34, 1994.

21. L. X. Dang. J. Chem. Phys., 100:9032-9034, 1994.

22. D. van Belle, M. Prevost, G. Lippens, and S. J. Wodak. ACS Symposium Series, 568:318-334, 1994.

23. B. Madan and B. Lee. Biophysical Chemistry, 51:279-289, 1994.

24. D. Sitkoff, K. A. Sharp, and B. Honig. Biophysical Chemistry, 51:397-409, 1994.

25. A. Holtzer. Biopolymers, 35:595-602, 1995.

26. S. K. Kumar, I. Szleifer, K. Sharp, P. J. Rossky, R. Friedman, and B. Honig. J. Phys. Chem., 99:8382-8391, 1995.

27. D. Horvath, D. van Belle, G. Lippens, and S. J. Wodak. J. Chem. Phys., 104:66796695, 1996.

28. T. Headgordon. Proc. Natl. Acad. Sci. USA, 92:8308-8312, 1995.

29. A. D. J. Haymet, K. A. T. Silverstein, and K. A. Dill. Faraday Discussions, 103:117$124,1996$.

30. S. Garde, G. Hummer, and M. E. Paulaitis. Faraday Discussions, 125-139, 1996.

31. K. A. Sharp, S. Kumar, P. J. Rossky, R. A. Friedman, and B. Honig. J. Phys. Chem., 100:14166-14177, 1996.

32. M. Prevost, I. T. Oliveira, J. P. Kocher, and S. J. Wodak. J. Phys. Chem., 100:27382743, 1996.

33. P. M. Wiggins. Physica A, 238:113-128, 1997.

34. D. Chandler, J. D. Weeks, and H. C. Andersen. Science, 220:787-794, 1983.

35. B. Widom. J. Chem. Phys., 39:2808-2812, 1963.

36. B. Widom. J. Phys. Chem., 86:869-872, 1982.

37. H. Reiss. J. Phys. Chem., 96:4736-4747, 1992.

38. B. Lee. Biopolymers, 31(8):993-1008, 1991.

39. F. M. Richards. Scientific American, 264(1):54, 1991.

40. R. A. Pierotti. J. Phys. Chem., 67:1840-1845, 1963.

41. R. A. Pierotti. Chem. Rev., 76:717-726, 1976.

42. L. R. Pratt and D. Chandler. J. Chem. Phys., 67:3683-3704, 1977.

43. L. R. Pratt. Ann. Rev. Phys. Chem., 36:433, 1985. 
44. L. R. Pratt and D. Chandler. Methods in Enzymology, 127:48, 1985.

45. G. Hummer and S. Garde. Phys. Rev. Letts., 80:4193-4196, 1998.

46. J. Riordan. An Introduction to Combinatorial Analysis. Princeton University Press, Princeton, NJ, 1978.

47. J. E. Mayer and E. Montroll. J. Chem. Phys., 9:2-16, 1941.

48. H. Reiss, H. L. Frisch, and J. L. Lebowitz. J. Chem. Phys., 31:369-380, 1959.

49. N. G. van Kampen. Stochastic Processes in Physics and Chemistry. North-Holland, New York, 2nd edition, 1992. Section II.4.

50. H. C. Andersen. Volume 5 of Modern Theoretical Chemistry, Statistical Mechanics, Part A: Equilibrium Techniques. Plenum, New York, 1977.

51. J.-P. Hansen and I. R. McDonald. Theory of Simple Liquids. Academic Press, San Diego, CA, 2nd edition, 1991. Chapter 4.

52. R. Kubo. J. Phys. Soc. Japan, 17:1110-1120, 1962.

53. P. Carruthers. Phys. Rev. A, 43:2632-2639, 1991.

54. R. L. Graham, D. E. Knuth, and O. Patashnik. Concrete Mathematics. AddisonWesley, Reading, MA, 2ndmerely edition, 1994. Section 2.6.

55. J. E. Shore and R. W. Johnson. IEEE Transactions on Information Theory, 26(1):26-37, 1980.

56. D. Chandler. Phys. Rev. E, 48:2898-2905, 1993.

57. J. K. Percus. J. de Phys. IV, 3:49-57, 1993.

58. G. Hummer, S. Garde, A. E. Garcia, A. Pohorille, and L. R. Pratt. Proc. Nat. Acad. Sci. USA, 93:8951, 1996.

59. B. J. Berne. Proc. Nat. Acad. Sci. USA, 93:8880, 1996.

60. S. Garde, G. Hummer, A. E. Garcia, M. E. Paulaitis, and L. R. Pratt. Phys. Rev. Letts., 77:4966-4968, 1996.

61. L. R. Pratt. In Encyclopedia of Computational Chemistry. 1998. LA-UR-96-4478, (in press 1998).

62. W. Kauzmann. Nature (London), 325:763-764, 1987.

63. G. Hummer, S. Garde, A. E. Garcia, M. E. Paulaitis, and L. R. Pratt. Proc. Natl. Acad. Sci. USA, 95:1552-1555, 1998.

64. G. Hummer, S. Garde, A. E. García, M. E. Paulaitis, and L. R. Pratt. J. Phys. Chem. B, 1998. LA-UR-98-xxxx.

65. J. D. Weeks, R. L. B. Selinger, and J. Q. Broughton. Phys. Rev. Letts., 75:26942697, 1995.

66. J. D. Weeks, K. Vollmayr, and K. Katsov. Physica A, 244:461-475, 1997.

67. L. R. Pratt and R. A. LaViolette. Quasi-chemical theories of associated liquids. Molec. Phys., 1998. (in press).

68. M. D. Eldridge, P. A. Madden, and D. Frenkel. Nature, 365:35-37, 1993.

69. D. Frenkel. Physics World, 6:24-25, 1993.

70. D. Frenkel. J. Phys.: Condens. Matter, 6:A71-A78, 1994.

71. M. Dijkstra, D. Frenkel, and J. P. Hansen. J. Chem. Phys., 101:3179-3189, 1994.

72. M. Dijkstra and D. Frenkel. Phys. Rev. Letts., 72:298-300, 1994.

73. D. Frenkel. Future Generation Computer Systems, 10:207-212, 1994.

74. M. Dijkstra and R. Vanroij. Phys. Rev. E, 56:5594-5602, 1997.

75. G. E. Crooks and D. Chandler. Phys. Rev. E, 56:4217-4221, 1997.

76. H. C. Andersen. Ann. Rev. Phys. Chem., 26:145-166, 1975. 